Military Technical College, Kobry El-Kobbah, Cairo, Egypt $9^{\text {th }}$ International Conference

On Aerospace Sciences \& Aviation Technology

\title{
COMPARATIVE STUDY OF GLOBALSTAR AND CDMA IRIDIUM LEO SYSTEMS
}

\author{
HEBATALLAH M. MOURAD* ABD EL-AZIZ M. EL-BASIONI** SHERIF S. EMAM ${ }^{\star \star \star \star}$ \\ EMAD K. AL-HUSSAINI $I^{\star \star \star \star}$
}

\section{ABSTRACT}

The TDMA-Iridium and the CDMA GlobalStar are two of the main LEO satellite systems. Since the trend is towards CDMA due to its valuable advantages, a version of the TDMA-Iridium namely CDMA-Iridium is proposed. A comparison between GlobalStar and the proposed system is performed concerning the signal- tointerference ratio (SIR), the throughput of the Dense Traffic Satellite (DTS) and Sparse Traffic Satellite (STS) are considered. Adaptive Transmit Permission Control (ATPC) method is used to improve the throughput for both systems.

\section{KEYWORDS}

Low Earth Orbit Satellite Communication

\section{INTRODUCTION}

The LEO systems, while having the most important features of conventional Geostationary satellite communication systems, such as wide coverage area, direct radio path and flexibility of the network architecture, provide additional

Advantages for the global communication networks as small propagation delay and loss and high elevation angle in high altitudes [1]. The SIR and the throughput performance of Geostationary satellites are already existing in a lot of text books and papers [2-6]. However the study of LEO systems has two main difficulties: the nonuniformity of the traffic and the dynamics of the system since the satellites are in continuous motion with respect to the Earth station. These two problems wore investigated in $[7,8]$. It has also been recognized that CDMA offers random access

*Assistance professor, Dept. of Communication, Faulty of Engineering, Cairo University, Giza, Egypt.

** Lucent Technologies, Cairo Office.

*** PhD. Student.

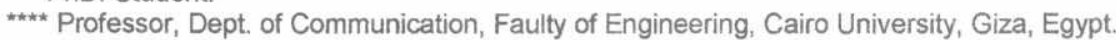


channel sharing with low delay, along with spread spectrum advantages such as immunity to external interference and jamming...etc[9]. Thus one of the best solutions to global personal communication network ( $\mathrm{PCN}$ ) is the use of CDMA-LEO satellites.

This paper follows the reported ideas in the previous mentioned paper to establish a comparison between the GlobalStar and the proposed system namely CDMA-Iridium system[10,11].

In section II the mathematical model for traffic nonuniformity is introduced. In section III, the SIR for both systems is investigated, the effect of satellite position on the traffic nonuniformity on SIR for these systems are discussed. In section IV, the traffic assignment control method is applied. Section $V$ compares their throughput. In section VI, ATPC method is employed. Section VII, includes conclusions.

\section{THE SYSTEM MODEL}

The two dimensional system model is shown in Fig.1. In this model [10], an area on the Earth is represented by an arc. In this figure, we distinguish between the coverage area of a satellite and the interference area of it. The coverage area is specified by the minimum value of the elevation angle, $\theta_{\min }$ that an Earth station is assumed to be able to access to the satellite while the interference area is determined by the final line of sight of that satellite. The service area is defined as a limited area within a coverage area where users can connect to the satellite. The double coverage area is an area commonly located between two or more adjacent coverage areas. It should be noted that if an earth station lies in the interference area but out of the coverage area of a satellite, it would not be allowed to connect to that satellite, but still its signal reaches that satellite as interference. In order to analyze the influence of the nonuniformity in traffic, we focus on a series of three satellites and their users. Assume that a total numbers of users, $N_{4}$ are distributed randomly in a given area the size of which is equal to the service areas of three adjacent satellites and their interference areas. For a LEO satellite system in which the satellites have the altitude $h(\mathrm{~km})$, the geometric interference limit for each of them is determined by the following angle.

$$
\beta_{I}=\cos ^{-1}\left(\frac{R}{R+h}\right)
$$

Were $R$ is the average radius of the earth. The distribution of the users is assumed to have the following function $[8,10]$ :

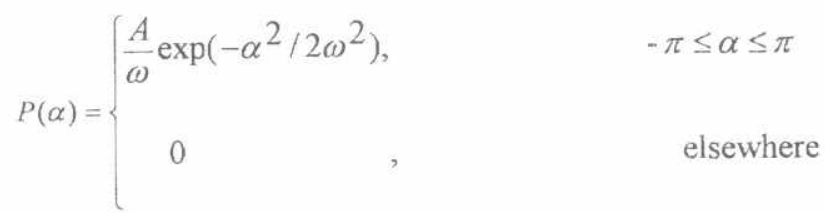


Where $\alpha$ is the relative location of a user, measured by the angle at the center of the earth; $\omega$ is the parameter representing uniformity in traffic; $N_{s}$ is the number of satellites in each orbit; and $A$ is a factor that makes the total traffic load in the area fixed and equal to $N_{u}$, when changing $\omega$ or $N_{s}$, and equals to

$$
A=N u / \int_{-3 \pi / N s}^{3 \pi / N s}\left[\exp \left(-\alpha^{2} / 2 \omega^{2}\right) / \omega\right] d \alpha
$$

$B_{i}$ is the angular position of the $i$-th satellite. In this paper two LEO systems are considered, the GlobalStar located at a height of $1400 \mathrm{~km}$, and contains 6 satellites in each orbit, $\theta_{\min }=5^{\circ}$, and a proposed system which will be located at a height of 800 $\mathrm{km}$ and contains 11 satellites in each orbit, $\theta_{\min }=10^{\circ}$. The total number of users $\left(N_{u}\right)$ is assumed to be 100 distributed in the area for both systems

The ratio of the traffic loads of two adjacent natural service areas, when the peak of the traffic is located at the origin, as shown in Fig.2, is defined as:

$$
T R=\int_{-\pi / N s}^{\pi / N_{s}} P(\alpha) d \alpha / \int_{\pi / N s}^{3 \pi / N s} P(\alpha) d \alpha
$$

The traffic ratios for the two systems under consideration are shown in Fig. 2. We see that the traffic ratio for $N_{s}=6$ is larger than for $N_{s}=11$ for any $\omega$. For $\omega=0.5, \quad N_{s}=11$, the average number of users of DTS is equal to 45 , the average number of users of STS is equal to 25 , the traffic ratio is nearly equal to 1.8 . For $\omega=0.5, N_{s}=6$, the average number of users of DTS is equal to 70 , the average number of users of STS is equal to 15 , the traffic ratio is nearly equal to 4.7 , i.e the non-uniformity of the traffic is more pronounced in the Globalstar case. Furthermore, the traffic ratio tends to one, i.e approaches the uniform case at $w$ equals approximately 1.5 and 2 for the CDMA-Iridium and the Globalstar respectively.

\section{SIGNAL-TO-INTERFERENCE RATIO(SIR)}

Assuming that all satellites have the same designed power levels. The SIR of the ith and $(i+1)$ th can be written as: $[5,12,13]$.

$$
\operatorname{SIR}_{i}=0.5\left[\int_{0}^{\pi / N} P(\alpha) d \alpha+\int_{\pi / N_{s}}^{\beta I} P(\alpha) I_{i+1}^{2}(\alpha) I_{i}^{-2}(\alpha) d \alpha\right]^{-1}
$$


$S I R_{i+1}=\left[\int_{\pi / N_{s}}^{3 \pi / N_{s}} P(\alpha) d \alpha+\int_{2 \pi / N_{s}-\beta t}^{\pi / N_{s}} P(\alpha) I_{i}^{2}(\alpha) I_{i+1}^{-2}(\alpha) d \alpha \int_{3 \pi / N_{s}}^{2 \pi} P(\alpha) I_{i+2}^{2}(\alpha) I_{i+1}^{-2}(\alpha) d \alpha\right]^{-1}$

Where $I_{(\alpha)}$ represents the distance between the ith satellite and the Earth station at the angular offset $\alpha$

Because in a LEO satellite system the satellites are on nongeostationay orbits, they are in continuous motion, with relatively high ground speed. The changes in SIR characteristics according to the travel of satellites are examined. In this analysis, we assume that the satellites are on circular orbits which are usually used in LEO satellite system constellations and simplify the control of the system considerably.

Fig. 3 shows how the degree of traffic nonuniformity affects the performance of the system. in large traffic nonuniformity (i.e., small $\omega$ ), there are large differences between the signal qualities of the DTS and STS. We conclude that these differences are larger for the GlobalStar than the CDMA-Iridium, since the traffic ratio is higher for the first system. As $\omega$ increases, i.e approaching uniform traffic, the SIR for the dense and sparse satellites is approximately the same

An important point drawn from that figure is that for any of the two systems there are large variations in signal quality at each satellite when a nonuniform distribution of users exists. This phenomenon may be acceptable while the level of SIR is higher than a threshold that ensures an acceptable error rate; however, it means that the users of the communications system have to accept large tolerances in their service quality performance, even during short periods of time, which is not good behavior for a reliable communications system

Furthermore for small $\omega$ (i.e non-uniform case) in case of DTS, SIR, for $N_{s}=11$ is better than $N_{s}=6$, since the average number of users is smaller. However the situation is reversed for the STS for the same reasoning. Fig. 4 shows the changes of SIRfor the DTS and STS for both systems as a function of the position of the ith satellite. This figure supports the last two comments.

\section{TRAFFIC ASSIGNMENT CONTROL}

In the previous sections, natural service area configuration was assumed, i.e, equal receiving powers and equal service areas area required for all satellites. That configuration, although natural in the case of uniform traffic, no longer has merit when the nonuniform distribution of users is involved. A method that can change the size of service areas according to the offered traffic loads is proposed in [11]. In this method, namely the modified power control (MPC), the designed receiving powers of the satellites are not equal. The proposed method would control the size of service areas according to their local traffic loads, that is, the service areas with lighter traffic loads are expanded, and those with heavier traffic loads are decreased. The ratio of the desired receiving power of the DTS to that of its neighbors on both sides is.

$$
y=S_{i} / S_{i-1}=S_{i} / S_{i+1}
$$

Where $S_{i}, S_{i+1}, S_{i-1}$, are the designed receiving power level at the ith, (i+1)th, $(i-1)$ th, satellites respectively. 
Fig.5 shows that increasing y will improve the SIR performance of the DTS and deteriorates the SIR of the STS. In general we prefer to work at the value of $y$ that maintain the same SIR for the two adjacent satellites. This value is 1.2 for the CDMAIridium and 2.7 for the GlobalStar since we need to decrease the service area of the DTS furthermore for the second system since the traffic ratio is higher.

\section{THROUGHPUT RESULTS}

In the case of LEO satellite systems with non-uniform traffic distribution, the expected number of users and hence the composite packet arrival rate are different in each service area. Thus, we normalize the throughput for each satellite $\left(\zeta_{i, n o r m}\right)$ by the expected number of users in its service area, $E\{\mathrm{Ni}\}$. The normalized throughput for the ith satellite, is given by:

$$
\xi_{i, \text { norm }}=\frac{\xi_{\mathrm{i}}}{\mathrm{E}\{\mathrm{Ni}\}} \quad, \mathrm{i}=1,2, \ldots \ldots, \mathrm{Ns}
$$

Where $\xi_{i}$ is the expected number of successfully transmitted packets of the ith satellite. Assume that $c_{i}$ is the number of successful transmissions for the ith satellite among simultaneous $n_{i}$ packets transmitted from its service area when $m_{i}$ packets are sent from its interference area. In that case [4],

$$
P\left[C_{i}=c / n_{i}=n, m_{i}=m\right]=\left(\begin{array}{l}
n \\
c
\end{array}\right) P_{c, i}^{c}(n, m)\left[1-p_{c, t}(n, m)\right]^{n-c}
$$

Where $P[A]$ define the occurrence probability of event $A$, and $P_{c, i}(n, m)$ is the probability of success for a packet in the presence of $n$ and $m$ packets in the service area and in the interference area of the ith satellite, respectively. The throughput for this case is the expected number of successful transmissions to the ith satellite, is given by

$$
\xi_{i}\left(n_{i}, m_{i}\right)=E\left[c_{i} \mid n_{i}, m_{i}\right]
$$

and it can be written in the form

$$
\xi_{\mathrm{i}}\left(\mathrm{n}_{\mathrm{i}}, \mathrm{m}_{\mathrm{i}}\right)=\sum_{\mathrm{c}=0}^{n_{i}} \mathrm{c}\left(\begin{array}{c}
\mathrm{n}_{\mathrm{i}} \\
\mathrm{c}
\end{array}\right) \mathrm{P}_{\mathrm{c}, \mathrm{i}}^{\mathrm{c}}\left(\mathrm{n}_{\mathrm{i}}, \mathrm{m}_{\mathrm{i}}\right)\left[1-\mathrm{P}_{\mathrm{c}, \mathrm{i}}\left(n_{i}, m_{i}\right)\right]^{\mathrm{n}_{\mathrm{i}}-\mathrm{c}}
$$

The summation equals $n_{i} . P_{c, l}(n, m)$, then,

$$
\xi_{i}\left(n_{i}, m_{i}\right)=n_{i} \cdot P_{c i}\left(n_{i}, m_{i}\right)
$$

To find the total throughput of the ith satellite, we should average for all possible values of $n_{i}$ and $m_{i}$. To calculate that average value, we also should consider the probability of $n_{i}$ and $m_{i}$ packets from a total $N_{u}$ users. Hence, the throughput of the ith satellite, $\xi_{i}$ is the expected value of $\xi_{i}\left(n_{i}, m_{i}\right)$, that is, 


$$
\begin{aligned}
& \xi_{\mathrm{i}}=\mathrm{E}\left\{\mathrm{n}_{\mathrm{i}}, \mathrm{m}_{\mathrm{i}}\right\}= \\
& \sum_{\mathrm{M}=1}^{\mathrm{N}_{\mathrm{u}}} \mathrm{P}\left[\mathrm{M}_{\mathrm{i}}=\mathrm{M}\right] \sum_{\mathrm{m}=1}^{\mathrm{M}} \mathrm{f}(\mathrm{m}, \mathrm{M}) \sum_{\mathrm{n}=1}^{\mathrm{m}} \mathrm{P}\left[\mathrm{n}_{\mathrm{i}}=\mathrm{n} / \mathrm{m}_{\mathrm{i}}=\mathrm{m}\right] \mathrm{nP}_{\mathrm{c}, \mathrm{i}}(n, m)
\end{aligned}
$$

Where $M_{i}$ is the number of users in the interference area, and $P\left[M_{i}=M\right]$, is the probability of $M$ users in the interference area of the ith satellite, that is,

$$
p\left[M_{i}=M\right]=\left(\begin{array}{c}
N_{u} u \\
M
\end{array}\right)\left[\int_{\beta_{i}-\beta_{i}}^{\beta_{i}+\beta_{i}} p(\alpha) d(\alpha)\right]^{M}\left[\begin{array}{c}
\beta_{i}+\beta_{I} \\
1-\int_{\beta_{i}-\beta_{l}} p(\alpha) d(\alpha)
\end{array}\right]^{N_{u}-M}
$$

where $f(m, M)$ is the distribution of the number of packets that are sent simultaneously to the channel which is assumed to be binomial with parameters $q$ and $M$. " $q$ " is the probability that each user sends a packet in each time slot. $P\left[n_{i}=n / m_{i}=m\right]$ is the conditional probability of $n$ packets in the service area of the ith satellite when there is information on existing $m$ packets in the interference area of that satellite[15]. We will define an important parameter in the throughput calculations namely, $\mathrm{K}(\mu)$, where $\mu$ is the average symbol energy to noise ratio at the satellite. This parameter denotes the ratio of power of interference to power of the desired signal at each satellite. Abass [11] shows that $\mathrm{K}\left(\mu_{\mathrm{c}}\right)+1$ denotes the possible number of simultaneous transmissions to each satellite in the absence of the background noise interference. Thus we named $\mathrm{K}\left(\mu_{0}\right)$ multiple access capability. This is often used in the case of spread spectrum systems, and it means that the transmission of a packet is successful if the level of SNR is greater than the threshold $\mu_{c}$

In this section we evaluate the normalized throughput for both the CDMA-Iridium and the GlobalStar systems for the DTS and STS.

Fig.6 shows the normalized throughput performances as a function of the total offered traffic load, q. N $N_{U}$ for DTS and STS for a relatively nonuniform traffic case, $(\omega=0.5)$ in which the average number of users of the DTS is about twice and five times of the STS users, for $\mathrm{Ns}=11$ and $\mathrm{Ns}=6$, respectively. From the figure, two facts can be pointed out: The first observation is the large difference in the performances of the DTS and the STS in the presence of nonuniform traffic for large offered traffic load for the same value of multiple access capability $\mathrm{K}\left(\mu_{\mathrm{c}}\right)$, since STS serves smaller number of users. This difference is large in case of GlobalStar since its traffic ratio is higher. For the same reason, the maximum GlobalStar DTS throughput occurs at lower traffic load and drops from a value of 0.7 to 0.5 approximately. The second observation is due to the large interference area compared with the service area. Therefore, there is a large interference power from the users of DTS and the performance of STS degrades if the multiple access capability is not so large compared to the expected number of users. This result is shown in Fig. 7 and Fig. 8.

Note that the expected number of users in the service area of the STS is about onehalf of those in the service area of the DTS in case of Ns=11, while it is one-fifth in case of $\mathrm{Ns}=6$. The difference in performance of the DTS and STS becomes larger in 
case of $\mathrm{Ns}=6$. Furthermore the expected number of users of STS for $N_{S}=11$ is 25 while it is 15 for $N_{S}=6$.

\section{ADAPTIVE TRANSMIT PERMISSION CONTROL (ATPC)}

As shown in the preceding section, the probability of the packet success or capture probability for a given packet decreases as the number of simultaneous transmissions increases. In the ATPC method [16], the capture probability is improved by avoiding the transmissions from the users with relatively high propagation loss to their connecting satellites. These users, which are in the marginal areas, give equal-power interference to the local satellite and high power interference to the neighbor satellites and hence the elimination of their interference is of main role in the performance improvement. By applying this method, the radius of the service area of each satellite will be reduced by a factor $\rho$, where $0 \leq \rho \leq 1$. The selection of $\rho$ according to the change in the offered traffic load of individual satellites is considered, the decision is not common for all satellites and is performed by each one and is valid for that satellite merely. The statistics of the traffic load are used for determining the optimum value for $\rho$. The optimum value for $\rho$ in this method is the value that makes the throughput of each satellite maximum. This makes the satellite with lighter traffic loads select larger values for $\rho$. After this method is applied, the service area of the satellite with the higher traffic load becomes smaller than that of the satellite with the lighter traffic load. In this method we improve the throughput of each satellite separately; hence, the method exhibits better total throughput at the whole range of the offered traffic load In other words, in the ATPC method, we assign a traffic load to each satellite near to the traffic level that can be serviced by that satellite. This is the reason for its better performance. Let us compare the performance of the system without the ATPC method with the ones employing ATPC methods. Fig. 9 and 10 show that employing the adaptive method maintains good performance at light offered traffic load by disabling the ATPC (i.e, selecting $\rho=1$ ) and improves the throughput at higher offered traffic loads by gradually decreasing the value of the $\rho$.

\section{CONCLUSIONS}

From the previous comparative study, we conclude that the non-uniformity of the traffic is more in case of the GlobalStar than the proposed system (CDMA-Iridium). The traffic ratio is 4.7 compared to 1.8 . This leads to several conciusions. The first one is larger difference in SIR between the DTS and STS in non-uniform traffic in case of GlobalStar (6 dB compared to $2 \mathrm{~dB}$ ). The second one is that when applying MPC, a higher ratio of $y$ is needed in order to get the same SIR for DTS and STS. The same conclusions were reached concerning the throughput. Thus the need for TPC is more essential in case of the GlobalStar, since the discrepancy between the SIR or throughput of the DTS and the STS is more pronounced. 


\section{References}

[1] M. Katayama, A. Ogawa, and N. Morinage," Satellite communication systems with low earth orbits and the effect of Doppler shift," IEICE Trans., vol. J67-BII, no. 5, 1993, PP. 382-390

[2] Adams, W. S., and L. Rider, "Circular Polar Constellations Providing Continuous Single or Multiple Coverage Above a Specified Latitude,"J Astronaut. Sci., Vol. 35,1987.

[3] Ananasso, F., and F. D. Priscoli, "The Role of Satellite in Personal Communication Services," IEEE J. Select. Areas Commun., Vol. 13, No. 2 , 1995.

[4] Global Mobile Communications Systems Under Development, Washington, DC: Wishington Business, Oct. 18, 1993

[5] Roddy, D., Satellite Communications, Englewood Cliffs, NJ: Prentice-Hall, 1989

[6] Vatalaro, F., et al., "Analysis of LEO, MEO, and GEO Global Mobile Satellite Systems in the Prence of Interference and Fading,"IEEE J. Select. Areas Commun., Vol. 13, No. 2, 1995 pp. 291-300.

[7] A. Jamalipour, M. Katayama, T. Yamazato, and A. Ogawa,"a performance analysis on the effects traffic nonuniformity in low earth orbit satellite communication systems," Proc. $16^{\text {th }}$ Symp. Inform. Theory. Applicat. (SITA'93) (Japan), Vol. 1, 1993, PP. 203-206.

[8] A. Jamalipour, M. Katayama, T. Yamazato, and A. Ogawa, "Signal-tointereference of CDMA in low earth orbital satellite communication systems with nonuniform traffic distribution "Proc. IEEE GLOBE-COM'94, San Francisco, CA, 1994, PP. 1748-1752

[9] R. E. Kahn, S. A. Gronemeyer, J. Burchfiel, and R. C. Kunzelman,"Advances in packet radio technology," proc. IEEE, vol. 66,1978, pp. 1468-1496.

[10] A. Jamalipour et al., "performance of an integrated voice/Data system in non-uniform traffic low earth orbit satellite communication systems, "IEEEJ. Select. Areas Commun., Vol. 13, No. 2, 1995, PP. 465-473

[11] A. Jamalipour, and A. Ogawa, "Packet Admission Control in a Direct Sequence Spread-Spectrum LEO Satellite Commun. Network, "IEEE J. Select. Areas Commun., Vol. 15, No. 8, 1997, PP. 1649-1656.

[12] Pritchard, W. L., H. G. Suyderhoud, and R. A. Neison, Satellite Communication Engineering, $2^{\text {nd }} \mathrm{ed}$, Englewood Cliffs, NJ: Prentice-Hall, 1987.

[13] Werner, M., et al., "Analysis of System Parameters for LEO/ICO-Satellite Communication Networks," IEEE J. Select. Areas Commun., Vol. 13, No. 2 1995, pp.371-381

[14] Proakis, J., Digitial Communications, $2^{\text {nd }}$ ed., New York: McGraw-Hill, 1989

[15] Pursley, M. B., "Performance Evaluation for Phase-Coded-Spread-Spectrum Multiple Access Communication Part 1: System Analysis," IEEE Trans. Commun., Vol. COM-25, No. 8, 1977, pp.795-799.

[16] A. Jamalipour et al., "Transmit permission control on spread Aloha packets in LEO satellite systems," IEEE J. Select. Areas Commun., vol.14, 1996, pp. 1748-1757. 


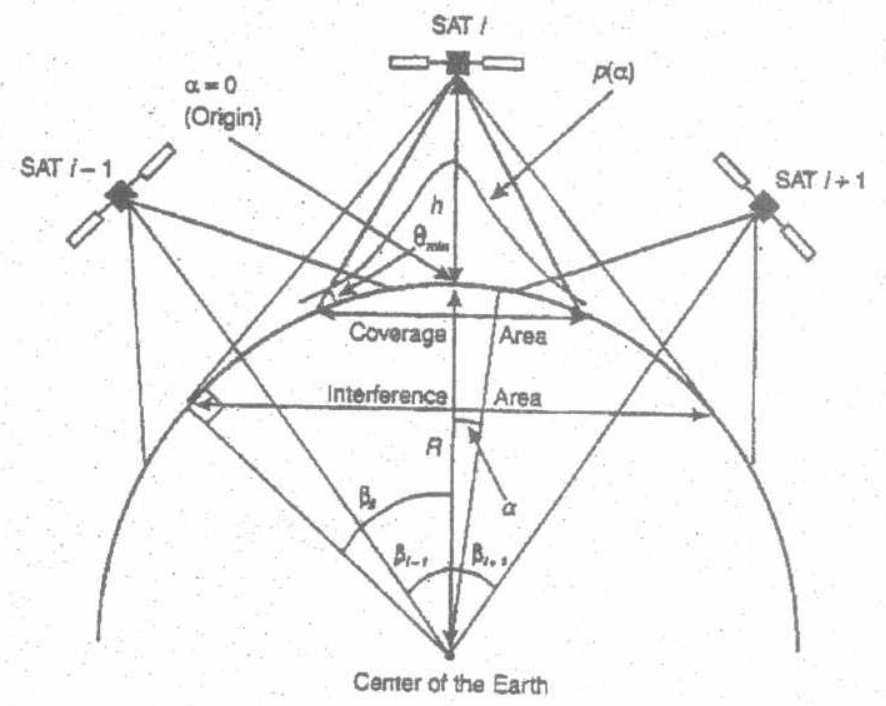

Fig.1. The System model 


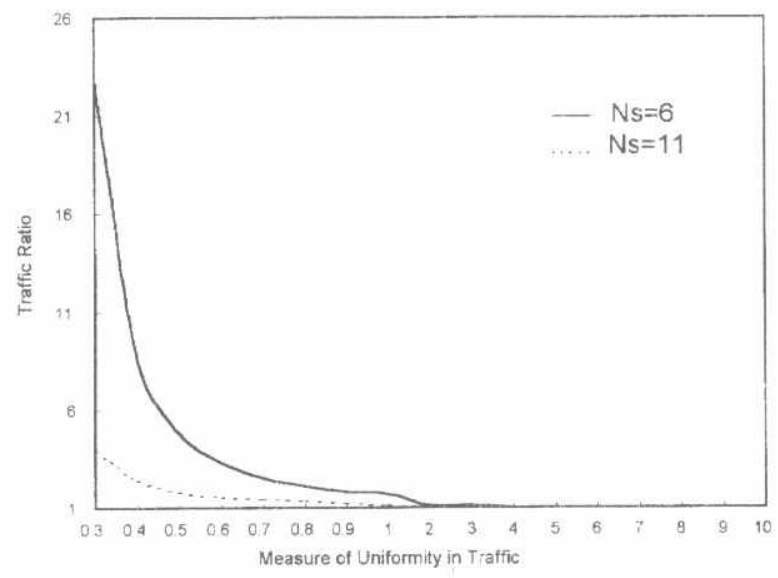

Fig.2 Ratio of traffic in the service area of two adjacent satellites (the first one is over the traffic peak) 


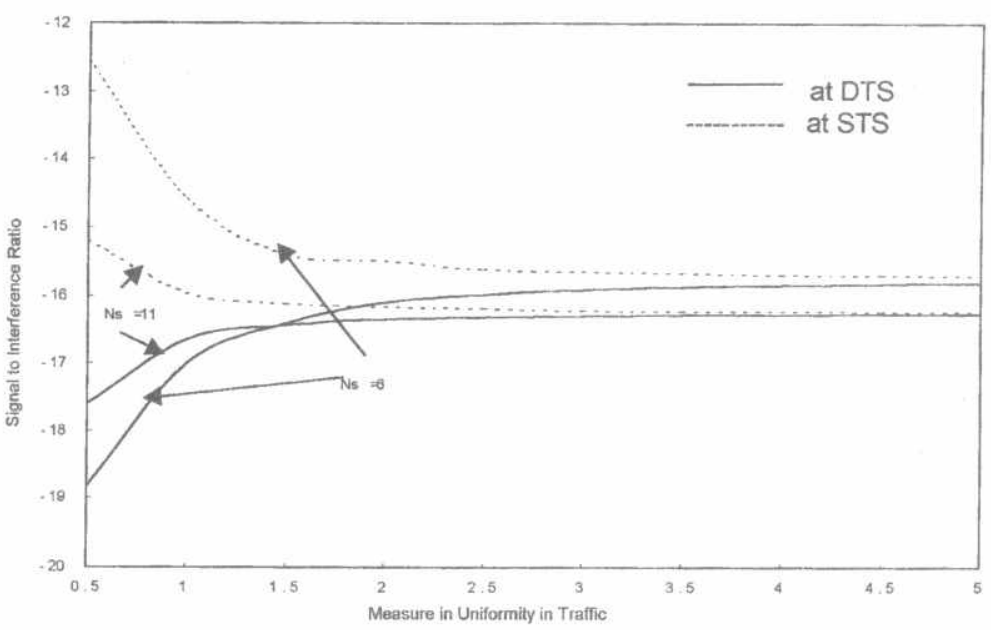

Fig. 3 SIR characteristics at DTS and STS, for $N_{s}=11,6$

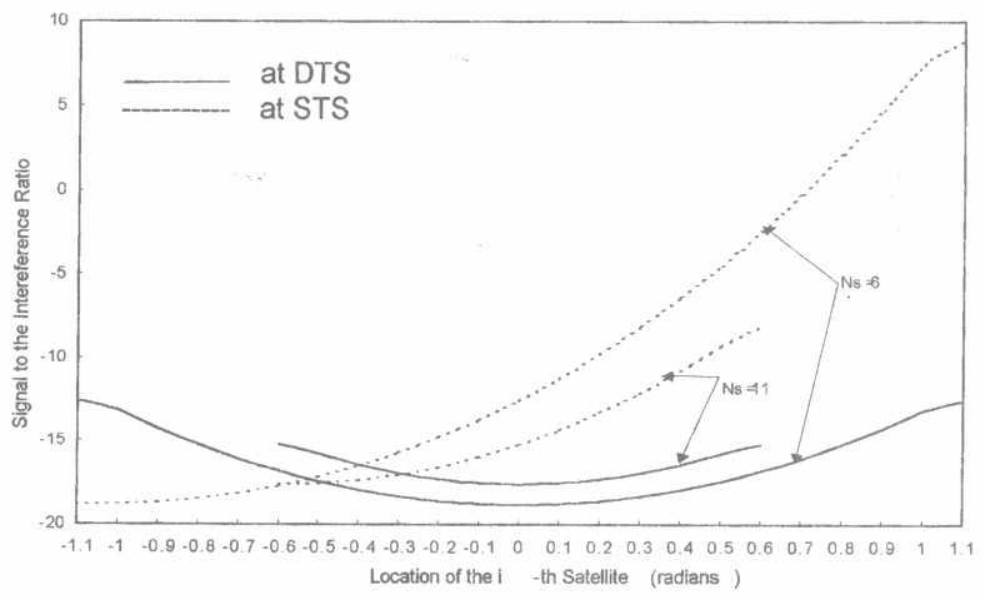

Fig. 4 Changes in SIR characteristics of two neighboring satellites as a function of their angular locations, $\omega=0.5$ 


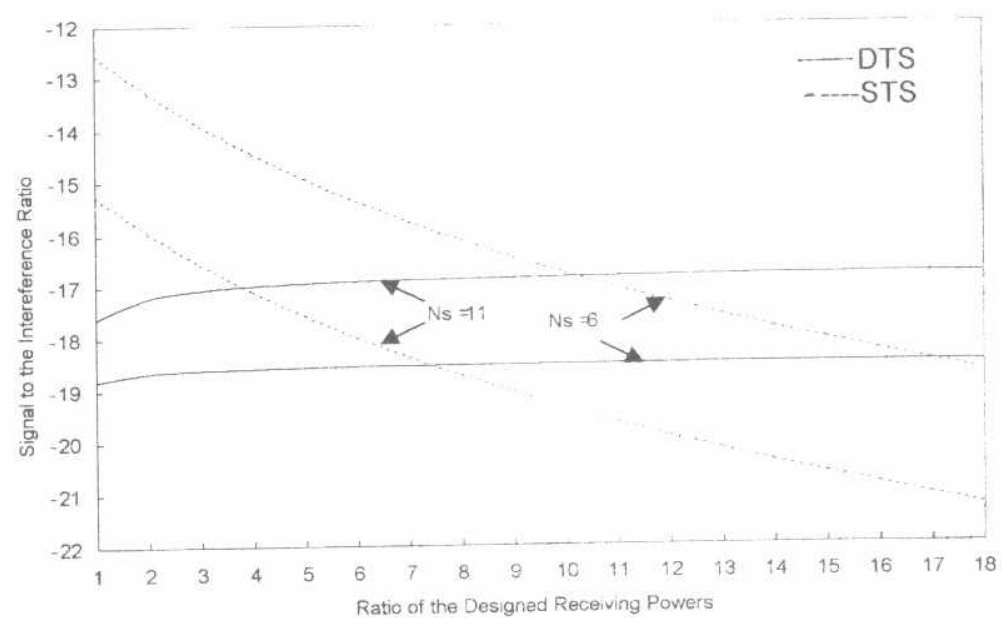

Fig. 5 Changes in SIR characteristics at DTS and STS as a result of a change in the ratio of the designed receiving power levels at the satellites, $\omega=0.5$

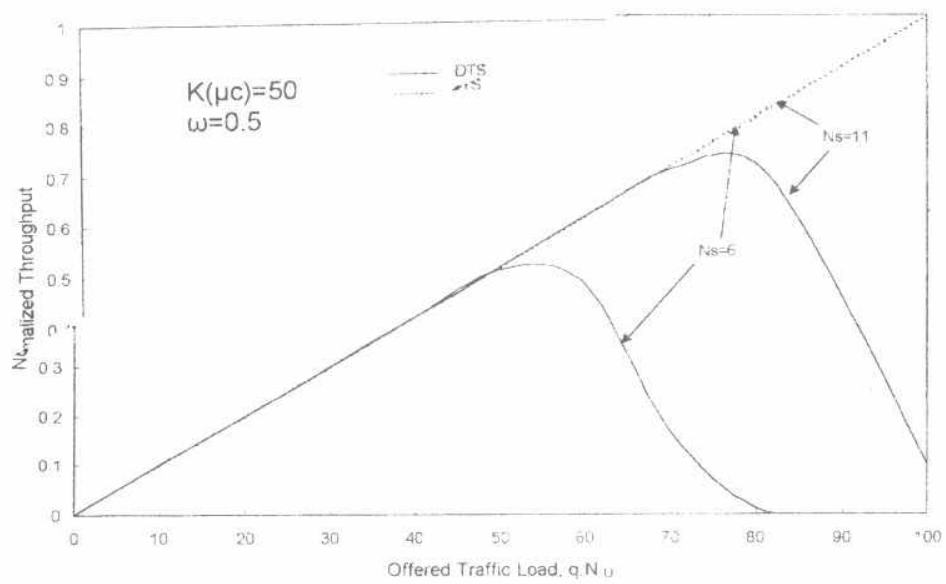

Fig.6 Normalized throughput as a function of total traffic load 


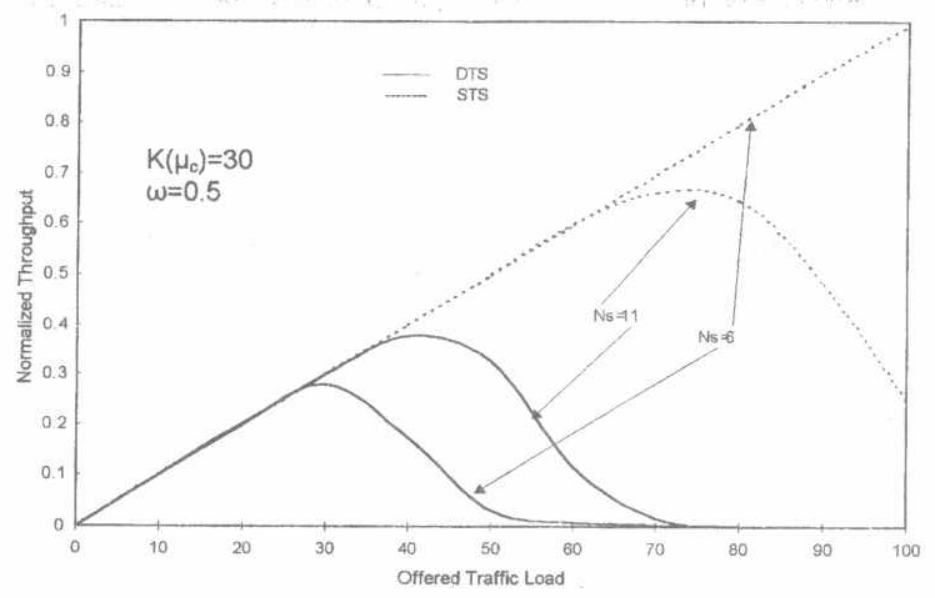

Fig.7 Normalized throughput as a function of total traffic load

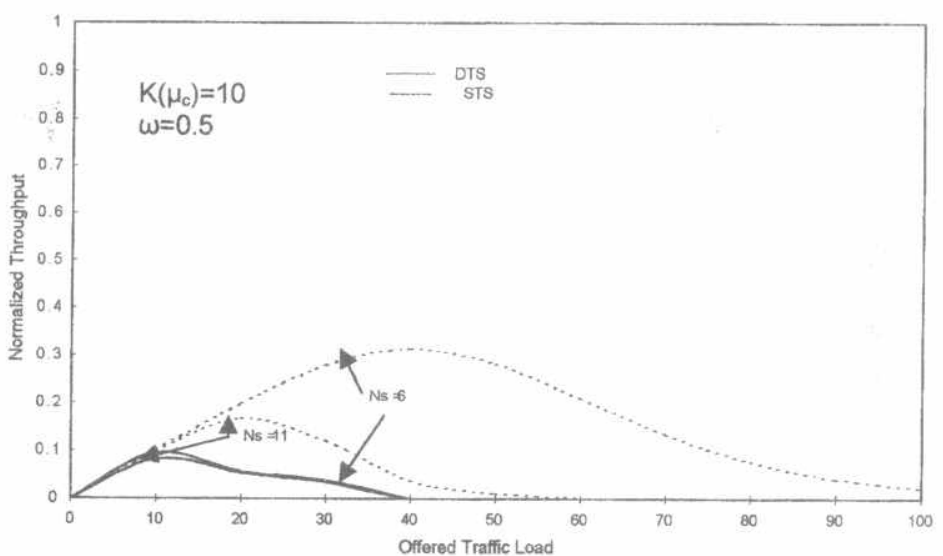

Fig.8 Normalized throughput as a function of total traffic load 


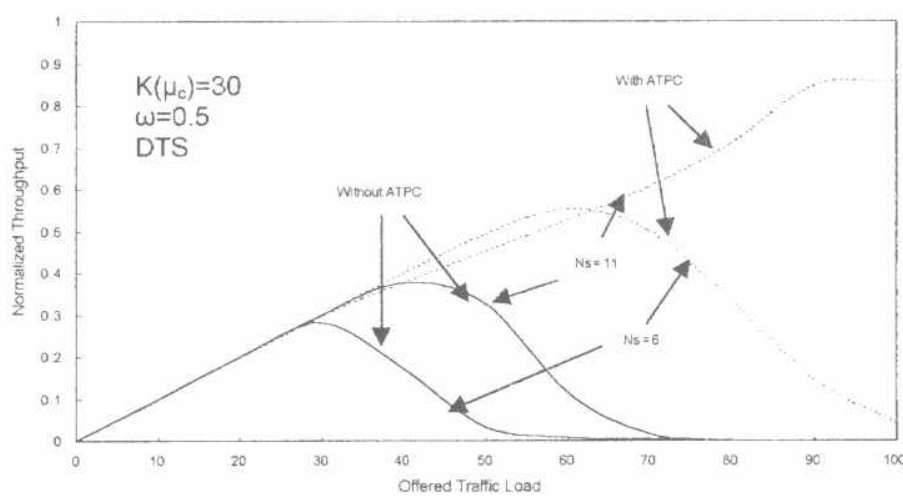

Fig. 9 Effect of the ATPC on the throughput performance of the satellite over the dense traffic area

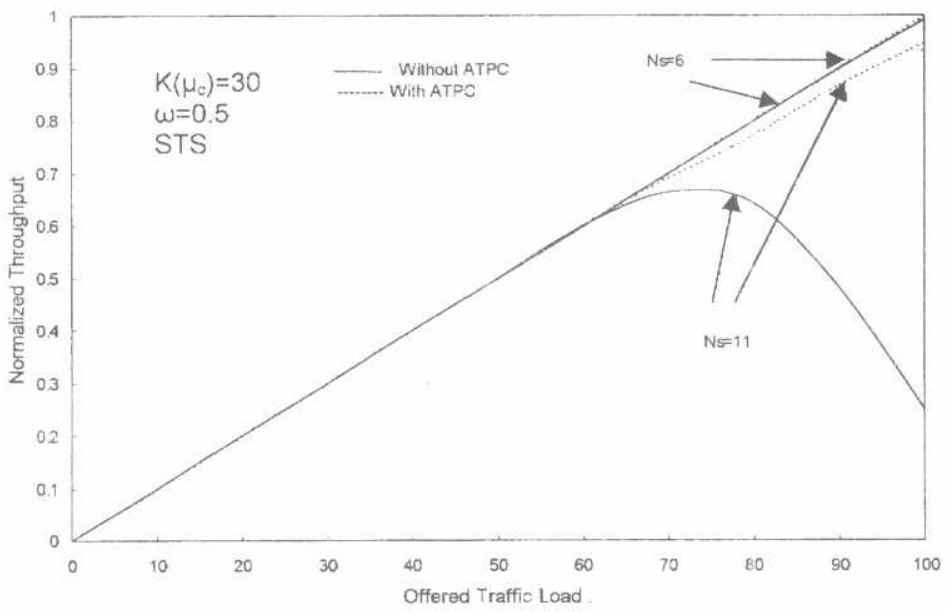

Fig. 10 Effect of the ATPC on the throughput performance of the satellite over the sparse traffic area 